\title{
ON THEOREMS CONNECTING THE LAPLACE TRANSFORM AND A GENERALIZED FRACTIONAL INTEGRAL OPERATOR
}

\author{
K. C. GUPTA, S. P. GOYAL AND TARIQ O. SALIM
}

\begin{abstract}
The aim of the present paper is to establish two theorems connecting the Laplace transform and a certain class of generalized fractional integral operators involving a generalized polynomial set. These theorems provide usful extension and unification of a number of (known or new) results for vaious classes of fractional integral operators. Several interesting applications of the main theorems are also mentioned briefly.
\end{abstract}

\section{Introduction}

Agrawal and Chaubey ([2, p. 1155]; see also Srivastava and Manocha [15, p. 447, Problem 16]) studied the following general sequence of functions:

$$
\begin{aligned}
R_{n}^{(\alpha, \beta)}[x ; a, b, c, d ; p, q ; \gamma, \delta ; w(x)]= & \frac{\left(a x^{p}+b\right)^{-a}\left(c x^{q}+d\right)^{-\beta}}{K_{n} w(x)} \\
& T_{k, l}^{n}\left\{\left(a x^{p}+b\right)^{a+\gamma n}\left(c x^{q}+d\right)^{\beta+\delta n} w(x)\right\},
\end{aligned}
$$

with the differential operator $T_{k, l}$ being defined as

$$
T_{k, l} \equiv x^{l}\left(k+x D_{x}\right)
$$

where $D_{x}=d / d x$.

In (1.1), $\left\{K_{n}\right\}_{n=0}^{\infty}$ is a sequence of constants, and $w(x)$ is independent of $n$ and differentiable an arbitrary number of times. The definition (1.1) was motivated essentially by an earlier work of Srivastava and Panda [17] (see also [15, p. 434 Eq. (82)]).

On taking $w(x) \equiv 1, p=d=1, c=-\tau$ in (1.1) and replacing $\beta$ by $\beta / \tau$ therein, we arrive at the following polynomial set after making some obvious changes in parameters.

$$
\begin{aligned}
& S_{n}^{\alpha, \beta, \tau}[x ; r, c, q, A, B, k, l] \\
& =(A x+B)^{-\alpha}\left(1-\tau x^{r}\right)^{\beta / \tau} T_{k, l}^{n}\left[(A x+B)^{\alpha+q n}\left(1-\tau x^{r}\right)^{(\beta / \tau)+c n}\right]
\end{aligned}
$$

Received March 23, 1998, Revised May 15, 1998.

1991 Mathematics Subject Classification. 44A10, 33C30, 26 A33.

Key words and phrases. Laplace transform, general sequence of functins, generalized polynomial set, fractional integral operator, hypergeometric function, Fox's H-function. 
The aforementioned polynomial set has recently been studied by Raizada [9]. Note that while defining the polynomial set (1.3) we have omitted a redundant parameter $m$ from the definition of the polynomial set by Raizada.

The explicit form of the generalized polynomial set given by (1.3) is

$$
\begin{gathered}
S_{n}^{\alpha, \beta, \tau}[x ; r, c, q, A, B, k, l]=B^{\dot{q} n} x^{l n}\left(1-\tau x^{r}\right)^{c n} l^{n} \sum_{e, p, u, \nu} \\
\frac{(-1)^{p}(-\nu)_{u}(-p)_{e}(\alpha)_{p}}{u ! \nu ! e ! p !} \frac{(-\alpha-q n)_{e}}{(1-\alpha-p)_{e}}(-\beta / \tau-c n)_{\nu}\left(\frac{e+k+r u}{l}\right)_{n}\left(\frac{-\tau x^{r}}{1-\tau x^{\tau}}\right)^{\nu}\left(\frac{A x}{B}\right)^{p} \\
\cdot \sum_{e, p, u, \nu} \equiv \sum_{\nu=0}^{n} \sum_{u=0}^{\nu} \sum_{p=0}^{n} \sum_{e=0}^{p}
\end{gathered}
$$

Taking $\tau \rightarrow 0$ in (1.3) and (1.4), we get the following

$$
\begin{aligned}
& S_{n}^{\alpha, \beta, 0}[x ; r, q, A, B, k, l] \\
= & (A x+B)^{-\alpha} \exp \left(\beta x^{r}\right) T_{k, l}^{n}\left[(A x+B)^{\alpha+q n} \exp \left(-\beta x^{r}\right)\right] \\
= & \sum_{e, p, u, \nu} \phi(e, p, u, \nu) x^{w},
\end{aligned}
$$

where

$$
\begin{aligned}
\phi(e, p, u, \nu) & =B^{q n-p} l^{n} \frac{(-1)^{P}(-\nu)_{u}(-p)_{e}(\alpha)_{p}}{u ! \nu ! e ! p !} \frac{(-\alpha-q n)_{e}}{(1-\alpha-p)_{e}}\left(\frac{e+k+r u}{l}\right) A^{p} B^{\nu}, \\
\text { and } \quad w & =\ln +p+r \nu(p, \nu=0,1, \ldots, n)
\end{aligned}
$$

It may be remarked here that though the polynomial set defined by (1.3) is a special case of the general sequence of functions (1.1) yet the polynomial set under study is sufficiently general in nature and it unifies and extends a number of classical polynomials introduced and studied by various research workers such as Chatterjea [4], Gould and Hopper [5], Krall and Frink [7], Singh and Shrivastava [14], etc. Some of the special cases of (1.3) are given by Raizada in tabular form ([9, p. 65]; see also Saigo, Goyal and Saxena [12], Agrawal, Pareek and Saigo [3]). Moreover, the explicit series form similar to (1.4) may not be easily obtainable for the sequence of functions defined by (1.1).

In this paper, we shall study the following two fractional integral operators involving a generalized polynomial set defined by (1.3):

$$
\begin{gathered}
R[f(x)]=R_{r, c, q, A, B, k, l ; \rho, \sigma}^{z ; n)} \\
=h x^{-\eta-h \mu-1} \int_{0}^{x} t^{\eta}\left(x^{h}-t^{h}\right)^{\mu} S_{n}^{\alpha, \beta, \tau}\left[z\left(\frac{t^{h}}{x^{h}}\right)^{\rho}\left(1-\frac{t^{h}}{x^{h}}\right)^{\sigma} ; r, c, q, A, B, k, l\right] \theta\left(\frac{t}{x}\right) f(t) d t,
\end{gathered}
$$


and

$$
\begin{aligned}
& K[f(x)]=K_{r ; n, \alpha, \beta, \tau ; \mu, \delta, h}^{2 ; n}, f(x) \\
= & h x^{\delta} \int_{x}^{\infty} t^{-\delta-h \mu-1}\left(t^{h}-x^{h}\right)^{\mu} S_{n}^{\alpha, \beta, \tau}\left[z\left(\frac{t^{h}}{x^{h}}\right)^{\rho}\left(1-\frac{x^{h}}{t^{h}}\right)^{\sigma} ; r ; c, q, A, B, k, l\right] \varphi\left(\frac{x}{t}\right) f(t) d t,
\end{aligned}
$$

provided that the general $\theta$ and $\varphi$ functions and $f$ are so specified that the integrals (1.9) and (1.10) exist.

The main object of this paper is to establish two theorems connecting the fractional integral operators defined by (1.9) and (1.10) and Laplace transform defined by

$$
L\{f(x) ; s\}=\int_{0}^{\infty} e^{-s x} f(x) d x=F(s),
$$

provided that $\operatorname{Re}(s)>0$, and $f(x)$ is so specified that the integral (1.10) exists.

\section{Main Theorems}

Theorem 1 .

$$
\begin{aligned}
L\{R[f(x)] ; s\} & =L\left\{\begin{array}{c}
R ; n, \alpha, \beta, \tau ; \mu, \eta, h \\
r ; c, q, A, B, k, l, \rho, \sigma
\end{array} f(x) ; s\right\} \\
& =h \int_{0}^{\infty} t^{\eta} f(t) E_{1}(s, t) d t
\end{aligned}
$$

and

$$
\begin{aligned}
L\{K[f(x)] ; s\} & =L\left\{\begin{array}{c}
z ; n, \alpha, \beta, \tau ; \mu, \delta, h \\
r ; c, q, A, B, k, l, \rho, \sigma
\end{array} f(x) ; s\right\} \\
& =h \int_{0}^{\infty} t^{-\delta-h \mu-1} f(t) G_{1}(s, t) d t
\end{aligned}
$$

provided that $\operatorname{Re}(s)>0, \theta, \varphi$ and $f$ are so specified that the integrals (2.1) and (2.2) exist.

$$
\begin{array}{r}
E_{1}(s, t)=L\left\{x^{-\eta-h \mu-1}\left(x^{h}-t^{h}\right)^{\mu} S_{n}^{\alpha, \beta, \tau}\left[z\left(\frac{t^{h}}{x^{h}}\right)^{\rho}\left(1-\frac{t^{h}}{x^{h}}\right)^{\sigma} ; r, c, q, A, B, k, l\right]\right. \\
\left.\cdot \theta\left(\frac{t}{x}\right) H(x-t) ; s\right\},
\end{array}
$$

and

$$
\begin{array}{r}
G_{1}(s, t)=L\left\{x^{\delta}\left(t^{h}-x^{h}\right)^{\mu} S_{n}^{\alpha, \beta, \tau}\left[z\left(\frac{x^{h}}{t^{h}}\right)^{\rho}\left(1-\frac{x^{h}}{t^{h}}\right)^{\sigma} ; r, c, q, A, B, k, l\right]\right. \\
\left.\cdot \varphi\left(\frac{x}{t}\right) H(t-x) ; s\right\},
\end{array}
$$


where $H(x)$ is the Heaviside unit function.

Proof. Making use of the definitions (1.11) and (1.9), (2.1) yields

$$
\begin{aligned}
\text { L.H.S. of }(2.1)= & h \int_{0}^{\infty} e^{-s x} x^{-\eta-h \mu-1} \int_{0}^{x} t^{\eta}\left(x^{h}-t^{h}\right)^{\mu} \\
& S_{n}^{\alpha, \beta, \tau}\left[z\left(\frac{t^{h}}{x^{h}}\right)^{\rho}\left(1-\frac{t^{h}}{x^{h}}\right)^{\sigma} ; r, c, q, A, B, k, l\right] \theta\left(\frac{t}{x}\right) f(t) d t d x,
\end{aligned}
$$

Changing the order of integrations which is easily justified under the conditions stated, we obtain

$$
\begin{aligned}
\text { L.H.S. of }(2.1)= & h \int_{0}^{\infty} t^{\eta} f(t) \int_{t}^{\infty} e^{-s x} x^{-\eta-h \mu-1} \int_{0}^{x} t^{\eta}\left(x^{h}-t^{h}\right)^{\mu} \\
& S_{n}^{\alpha, \beta, \tau}\left[z\left(\frac{t^{h}}{x^{h}}\right)^{\rho}\left(1-\frac{t^{h}}{x^{h}}\right)^{\sigma} ; r, c, q, A, B, k, l\right] \theta\left(\frac{t}{x}\right) d t d x,
\end{aligned}
$$

Now making use of definition of Heaviside unit function, (2.6) easily yields formula (2.1). Similarly the second assertion (2.4) of Theorem 1 can be established.

Theorem 2. Let $F(s)=L\{f(x) ; s\}$ exists. Then

$$
\begin{aligned}
& R[F(s)]=R_{r ; c, q, A, \beta, \tau ; \mu, \eta, h}^{z ;} ;, l, \rho, \sigma \\
& r(s) \\
&=h s^{-\eta-h \mu-1} \int_{0}^{\infty} t^{\eta} f(x) G_{2}(x, s) d x,
\end{aligned}
$$

and

$$
\begin{aligned}
K[F(s)] & =K \begin{array}{l}
z ; n, \alpha, \beta, \tau ; \mu, \delta, h \\
r ; c, q, A, B, k, l, \rho, \sigma
\end{array} F(s) \\
& =h s^{\delta} \int_{0}^{\infty} f(x) E_{2}(x, s) d x
\end{aligned}
$$

provided that $\operatorname{Re}(s)>0, \theta, \varphi$ and $f$ are so specified that the integrals (2.7) and (2.8) exist,

$$
\begin{array}{r}
G_{2}(x, s)=L\left\{t^{\eta}\left(s^{h}-t^{h}\right)^{\mu} S_{n}^{\alpha, \beta, \tau}\left[z\left(\frac{t^{h}}{s^{h}}\right)^{\rho}\left(1-\frac{t^{h}}{s^{h}}\right)^{\sigma} ; r, c, q, A, B, k, l\right]\right. \\
\left.\cdot \theta\left(\frac{t}{s}\right) H(s-t) ; x\right\}
\end{array}
$$

and

$$
\begin{array}{r}
E_{2}(x, s)=K\left\{t^{-\delta-h \mu-1}\left(t^{h}-s^{h}\right)^{\mu} S_{n}^{\alpha, \beta, \tau}\left[z\left(\frac{s^{h}}{t^{h}}\right)^{\rho}\left(1-\frac{s^{h}}{t^{h}}\right)^{\sigma} ; r, c, q, A, B, k, l\right]\right. \\
\left.\cdot \varphi\left(\frac{s}{t}\right) H(t-s) ; x\right\},
\end{array}
$$


Proof. The proof of Theorem 2 is much akin to that of Theorem 1, and we omit the details involved.

\section{Applications}

To be specific, we shall assume throughout this paper that

$$
f(x)= \begin{cases}O\left(|x|^{\xi}\right), & |x| \rightarrow 0 \\ O\left(|x|^{-\zeta} e^{-v|x|},\right. & |x| \rightarrow \infty\end{cases}
$$

Due to the presence of the general $\theta$ and $\varphi$ functios in the kernels of the fractional integral operators (1.9) and (1.10), many applications can be established by replacing $\theta$ and $\varphi$ by the known elementary special functions of one or more variables or polynomials of general type. An interesting application is considered in this section by replacing $\theta$ and $\varphi$ functions by the Gaussian hypergeometric function as follows

$$
\begin{aligned}
& \theta(z)={ }_{2} F_{1}\left(\mu+\eta+1,-\lambda ; \mu+1 ; 1-z^{h}\right) ; \\
& \phi(z)={ }_{2} F_{1}\left(\mu+\delta+1,-\lambda ; \mu+1 ; 1-z^{h}\right)
\end{aligned}
$$

So, the fractional integral operators defined by (1.9) and (1.10) are written as

$$
\begin{aligned}
R[f(x)]= & \left.R \begin{array}{c}
z ; n, \alpha, \beta, \tau ; \mu, \eta, h, \lambda \\
r ; c, q, A, B, k, l, \rho, \sigma
\end{array}\right) \\
= & h x^{-\eta-h \mu-1} \int_{0}^{x} t^{\eta}\left(x^{h}-t^{h}\right)^{\mu} S_{n}^{\alpha, \beta, \tau}\left[z\left(\frac{t^{h}}{x^{h}}\right)^{\rho}\left(1-\frac{t^{h}}{x^{h}}\right)^{\sigma} ; r, c, q, A, B, k, l\right] \\
& \cdot{ }_{2} F_{1}\left(\mu+\eta+1,-\lambda ; \mu+1 ; 1-\frac{t^{h}}{x^{h}}\right) f(t) d t
\end{aligned}
$$

It is easy to verify that this operator exists if

(i) $h>0, \rho, \sigma$ are non negative real numbers (not both zero simultaneously)

(ii) $h \operatorname{Re}(\mu)+h \sigma(\ell n+p+r \nu)(b, \nu=0,1, \ldots, n)>-1$ and (iii) $\operatorname{Re}(\eta+\xi)+h \rho(\ell n+p+$ $r \nu)(p, \nu=0,1, \ldots, n)>\max \{0, \operatorname{Re}(\eta-\lambda)\}-1$, and

$$
\begin{aligned}
K[f(x)]= & R \begin{array}{l}
z ; n, \alpha, \beta, \tau ; \mu, \delta, h \lambda \\
r ; c, q, A, B, k, l, \rho, \sigma
\end{array}(x) \\
= & h x^{\delta} \int_{x}^{\infty} t^{-\delta-h \mu-1}\left(t^{h}-x^{h}\right)^{\mu} S_{n}^{\alpha, \beta, \tau} \cdot\left[\left(\frac{x^{h}}{t^{h}}\right)^{\rho}\left(1-\frac{x^{h}}{t^{h}}\right)^{\sigma} ; r, c, q, A, B, k, l\right] \\
& \cdot{ }_{2} F_{1}\left(\mu+\delta+1,-\lambda ; \mu+1 ; 1-\frac{x^{h}}{t^{h}}\right) f(t) d t
\end{aligned}
$$

which exists under sets of conditions (i) and (ii) mentioned with (3.4) and $\operatorname{Re}(v)>0$ or $\operatorname{Re}(v)=0$ and $\operatorname{Re}(\delta+\zeta)+\rho(\ell n+p+r \nu)(p, \nu=0,1, \ldots, n)>\min \{0, \operatorname{Re}(\lambda-\delta)\}$. 
Now, setting $\tau \rightarrow 0, h=1$ and $\sigma=0$, and replacing $\mu$ by $(\mu-1)$ in (3.4) and (3.5), we get

$$
\begin{aligned}
R^{*}[f(x)]= & R \begin{array}{c}
z ; n, \alpha, \beta, 0 ; \mu-1, \eta, 1 \lambda \\
r, q, A, B, k, l ; \quad \rho, 0
\end{array}(x) \\
= & x^{-\eta-\mu} \int_{0}^{\infty} t^{\eta}(x-t)^{\mu-1} S_{n}^{\alpha, \beta, 0}\left[z\left(\frac{t}{x}\right)^{\rho} ; r, q, A, B, k, l\right] \\
& \cdot{ }_{2} F_{1}\left(\mu+\eta,-\lambda ; \mu ; 1-\frac{t}{x}\right) f(t) d t
\end{aligned}
$$

$\rho>0, \operatorname{Re}(\mu)>0$ and $\operatorname{Re}(\eta+\xi)>\max \{0, \operatorname{Re}(\eta-\lambda)\}-1$, and

$$
\begin{aligned}
K^{*}[f(x)]= & \left.R \begin{array}{l}
z ; n, \alpha, \beta, 0 ; \mu-1, \delta, 1, \lambda \\
r, q, A, B, k, l, \quad \rho, 0
\end{array}\right) \\
= & x^{\delta} \int_{x}^{\infty} t^{-\delta-\mu}(t-x)^{\mu-1} S_{n}^{\alpha, \beta, 0}\left[z\left(\frac{x}{t}\right)^{\rho} ; r, q, A, B, k, l\right] \\
& \cdot{ }_{2} F_{1}\left(\mu+\delta,-\lambda ; \mu ; 1-\frac{x}{t}\right) f(t) d t
\end{aligned}
$$

$\rho>0, \operatorname{Re}(\mu)>0$ and $\operatorname{Re}(v)>0$ (or $\operatorname{Re}(v)=0$ and $\operatorname{Re}(\delta+\xi)>\min \{0, \operatorname{Re}(\lambda-\delta)\}$.

If further, we set $n=q=k=B=0$ and $A=l=r=1$, then the generalized polynomial set reduces to unity, and thus (3.6) and (3.7) can be written as

$$
\begin{aligned}
R^{*}[f(x)] & =x^{-\eta-\mu} \int_{0}^{x} t^{\eta}(x-t)^{\mu-1} F_{1}\left(\mu+\eta,-\lambda, \mu ; 1-\frac{t}{x}\right) f(t) d t \\
& =\Gamma(\mu) I_{0}^{\mu, \eta, \lambda} x^{\eta} f(x) ; \\
K^{*}[f(x)] & =x^{\delta} \int_{x}^{\infty} t^{\delta-\mu}(t-x)^{\mu-1}{ }_{2} F_{1}\left(\mu+\delta,-\lambda, \mu ; 1-\frac{x}{t}\right) f(t) d t \\
& =\Gamma(\mu) x^{\delta} J_{x \infty}^{\mu, \delta, \lambda} f(x)
\end{aligned}
$$

where $I_{0}^{\mu, \eta, \lambda}$ and $J_{x}^{\mu, \delta, \lambda}$ are the fractional integral operators studied by Saigo [10]. The operator $I_{0}^{\mu, \eta, \lambda}$ contains both the Riemann-Liouville and the Erdelyi-Kober fractional integral operators, and the operator $J_{x \infty}^{\mu, \delta, \lambda}$ contains both the Weyl and the corresponding Erdelyi-Kober fractional integral operators as special cases (see, e.g., [18, p.2]).

Another application can be considered by replacing $\theta$ and $\varphi$ functions in (1.9) and (1.10) by Fox's H-function as follows

$$
\theta(z)=\varphi(z)=H_{P, Q}^{M, N}\left[y\left(z^{h}\right)^{\rho^{\prime}}\left(1-z^{h}\right)^{\sigma^{\prime}} \mid \begin{array}{c}
\left(a_{j}, A_{j}\right)_{1, P} \\
\left(b_{j}, B_{j}\right)_{1, Q}
\end{array}\right]
$$


for details of this function, one can refer to Srivastava, Gupta and Goyal [16, pp. 83-85]. Thus, the fractional integral operators (1.9) and (1.10) can be written as

$$
\begin{aligned}
R^{* *}[f(x)]= & \left.R \begin{array}{l}
z, y ; n, \alpha, \beta, \tau ; \mu, \eta, h ; M, N \\
r, c, q, A, B, k, l ; \rho, \rho^{\prime}, \sigma, \sigma^{\prime} ; P, Q
\end{array}\right\} \\
= & h x^{-\eta-h \mu-1} \int_{0}^{x} t^{\eta}\left(x^{h}-t^{h}\right)^{\mu} S_{n}^{\alpha, \beta, \tau}\left[z\left(\frac{t^{h}}{x^{h}}\right)^{\rho}\left(1-\frac{t^{h}}{x^{h}}\right)^{\sigma} ; r, c, q, A, B, k, l\right] \\
& H_{P, Q}^{M, N}\left[y\left(\frac{t^{h}}{x^{h}}\right)^{\rho^{\prime}}\left(1-\frac{t^{h}}{x^{h}}\right)^{\sigma^{\prime}} \mid \begin{array}{l}
\left(a_{j}, A_{j}\right)_{1, P} \\
\left(b_{j}, B_{j}\right)_{1, Q}
\end{array}\right] f(t) d t,
\end{aligned}
$$

and

$$
\begin{aligned}
K^{* *}[f(x)]= & \left.R \begin{array}{l}
z, y ; n, \alpha, \beta, \tau ; \mu, \delta, h ; M, N \\
r, c, q, A, B, k, l ; \rho, \rho^{\prime}, \sigma, \sigma ; P, Q
\end{array}\right\} \\
= & h x^{\delta} \int_{x}^{\infty} t^{-\delta-h \mu-1}\left(t^{h}-x^{h}\right)^{\mu} S_{n}^{\alpha, \beta, \tau}\left[z\left(\frac{x^{h}}{t^{h}}\right)^{\rho}\left(1-\frac{x^{h}}{t^{h}}\right)^{\sigma} ; r, c, q, A, B, k, l\right] \\
& H_{P, Q}^{M, N}\left[y\left(\frac{x^{h}}{t^{h}}\right)^{\rho^{\prime}}\left(1-\frac{x^{h}}{t^{h}}\right)^{\sigma^{\prime}} \mid \begin{array}{c}
\left(a_{j}, A_{j}\right)_{1, P} \\
\left(b_{j}, B_{j}\right)_{1, Q}
\end{array}\right] f(t) d t
\end{aligned}
$$

and for conditions of validity of these operators, we refer to Srivastava, Gupta and Goyal [16, pp. 39-40].

Further, if we reduce the generalized polynomial set in (3.11) and (3.12) to unity, we get the fractional integral operators studied by Saxena and Kumbat [13].

Also, the fractional integral operators studied by Gupta and Soni [6] are special cases of our operators (1.9) and (1.10).

Now, let us assume that the Laplace transform of $x^{\gamma} R^{*}[f(x)]$ exists, where $R^{*}[f(x)]$ is defined by (3.6), then by applying (1.6) and changing order of summation and integration, we get

$$
\begin{array}{r}
L\left\{x^{\gamma} R^{*}[f(x)] ; s\right\}=\sum_{e, p, u, \nu} \phi(e, p, u, \nu) z^{w} \int_{0}^{\infty} f(t) t^{\rho w+\eta} \\
\int_{t}^{\infty} e^{-s x}(x-t)^{\mu-1} x^{\gamma-\rho w-\eta-\mu}{ }_{2} F_{1}\left(\mu+\eta,-\lambda ; \mu ; 1-\frac{t}{x}\right) d x d t
\end{array}
$$

and making use of the formula

$$
{ }_{2} F_{1}(a, b ; c ; z)=(1-z)^{-a}{ }_{2} F_{1}\left(a, c-b ; c ; \frac{z}{z-1}\right)
$$

we get

$$
\begin{aligned}
L\left\{x^{\gamma} R^{*}[f(x)] ; s\right\}= & \sum_{e, p, u, \nu} \phi(e, p, u, \nu) z^{w} \int_{0}^{\infty} f(t) t^{\rho w-\mu} \int_{\dot{t}}^{\infty} e^{-s x} \\
& (x-t)^{\mu-1} x^{\gamma-\rho w}{ }_{2} F_{1}\left(\mu+\eta, \mu+\lambda ; 1-\frac{x}{t}\right) d x d t
\end{aligned}
$$


and applying a known integral formula [11, p.18], we have

$$
L\left\{x^{\gamma} R^{*}[f(x)] ; s\right\}=\Gamma(\mu) \sum_{e, p, u, \nu} \phi(e, p, u, \nu) z^{w} \int_{0}^{\infty} t^{\rho w} f(t) U(\mu, \eta, \lambda, \gamma ; s, t) d t
$$

where

$$
\begin{aligned}
& U(\mu, \eta, \lambda, \gamma ; s, t)= t^{\gamma-\rho w} \frac{\Gamma(\eta-\gamma+\rho w) \Gamma(\lambda-\gamma+\rho w)}{\Gamma(\rho w-\gamma) \Gamma(\mu+\eta+\lambda-\gamma+\rho w)}{ }_{2} F_{2}(\gamma-\rho w+1, \\
&\gamma-\eta-\lambda-\mu+\rho w+1 ; \gamma-\eta-\rho w+1, \gamma-\lambda-\rho w+1 ;-s t) \\
&+s^{\eta+\rho w-\gamma} t^{\eta} \frac{\Gamma(\lambda-\eta) \Gamma(\gamma-\eta-\rho w)}{\Gamma(\mu+\lambda) \Gamma(-\eta)}{ }_{2} F_{2}(1-\mu-\lambda, \eta+1 ; \eta-\lambda+1,\eta-\gamma+\rho w+1 ;-s t) \\
&+s^{\lambda+\rho w-\gamma} t^{\lambda} \frac{\Gamma(\eta-\lambda) \Gamma(\gamma-\lambda-\rho w)}{\Gamma(\mu+\eta) \Gamma(-\lambda)}{ }_{2} F_{2}(1-\mu-\eta, \lambda+1 ; \lambda-\eta+1, \\
&\cdot \lambda-\gamma+\rho w+1 ;-s t)
\end{aligned}
$$

for $\operatorname{Re}(s)>0, \operatorname{Re}(\mu)>0$ and non integer values of $(\lambda-\eta),(\gamma-\eta-\rho w)$ and $(\gamma-\lambda-\rho w)$.

Also, if we assume that the Laplace transform of $x^{\gamma} K^{*}[f(s)]$ exists, where $K^{*}[f(x)]$ is defined by (3.7), then by applying a known formula [11, p.17], we get

$$
L\left\{x^{\gamma} K^{*}[f(x)] ; s\right\}=\Gamma(\mu) \sum_{e, p, u, \nu} \phi(e, p, u, \nu) z^{w} \int_{0}^{\infty} f(t) t^{\gamma} V(\mu, \delta, \lambda, \gamma ; s, t) d t
$$

where

$$
\begin{aligned}
V(\mu, \delta, \lambda, \gamma ; s, t)= & \frac{\Gamma(\gamma+\delta+\rho w+1) \Gamma(\gamma+\lambda+\rho w+1)}{\Gamma(\gamma+\rho w+1) \Gamma(\gamma+\delta+\lambda+\mu+\rho w+1)}{ }_{2} F_{2}(\gamma+\delta+\rho w+1, \\
& \gamma+\lambda+\rho w+1 ; \gamma+\rho w+1, \gamma+\delta+\lambda+\mu+\rho w+1 ;-s t)
\end{aligned}
$$

for $\operatorname{Re}(\gamma+\delta+\rho w)>\max (0, \operatorname{Re}(\delta-\lambda))-1$ and $\operatorname{Re}(s)>0$

Now, if $t$ is replaced by $x$ in (3.16) and (3.18), we get the following result contained in

Corollary 1. Let the Laplace transforms of $x^{\gamma} R^{*}[f(x)]$ and $x^{\gamma} K^{*}[f(x)]$ exist. Then

$$
L\left\{x^{\gamma} R^{*}[f(x)] ; s\right\}=\Gamma(\mu) \sum_{e, p, u, \nu} \phi(e, p, u, \nu) z^{w} \int_{0}^{\infty} x^{\rho w} f(x) U(\mu, \eta, \lambda, \gamma ; s, x) d x
$$

for $\operatorname{Re}(\mu)>0, \operatorname{Re}(s)>0$ and non integer values of $(\lambda-\eta),(\gamma-\eta-\rho w)$ and $(\gamma-\lambda-\rho w)$, and

$$
L\left\{x^{\gamma} K^{*}[f(x)] ; s\right\}=\Gamma(\mu) \sum_{e, p, u, \nu} \phi(e, p, u, \nu) z^{w} \int_{0}^{\infty} x^{\gamma} f(x) V(\mu, \delta, \lambda, \gamma ; s, x) d x
$$


for $\operatorname{Re}(s)>0$ and $\operatorname{Re}(\gamma+\delta+\rho w)>\max (0, \operatorname{Re}(\delta-\lambda))-1$ where $U$ and $V$ are defined by (3.17) and (3.19) respectively.

Also, following the same procedure which led to Corollary 1, we get from Theorem 2 , the result contained in

Corollary 2. Let $L\{f(x) ; s\}=F(s)$ exist. Then

$$
R^{*}\left[s^{\gamma} F(x)\right]=\Gamma(\mu) \sum_{e, p, u, \nu} \phi(e, p, u, \nu) z^{w} s^{\gamma} \int_{0}^{\infty} f(x) V(\mu, \eta, \lambda, \gamma ; x, s) d x
$$

for $\operatorname{Re}(s)>0$ and $\operatorname{Re}(\gamma+\eta+\rho w)>\max (0, \operatorname{Re}(\eta-\lambda))-1$, and

$$
K^{*}\left[s^{\gamma} F(x)\right]=\Gamma(\mu) \sum_{e, p, u, \nu} \phi(e, p, u, \nu) z^{w} s^{\rho w} \int_{0}^{\infty} f(x) U(\mu, \delta, \lambda, \gamma ; x, s) d x,
$$

for $\operatorname{Re}(\mu)>0, \operatorname{Re}(s)>0$ and non integer values of $(\lambda-\delta),(\gamma-\delta-\rho w)$ and $(\gamma-\lambda-\rho w)$ where $U$ and $V$ are as defined by (3.17) and (3.19) respectively.

Note that if the generalized polynomial set involved in the fractional integral operators $R^{*}$ and $K^{*}$ is reduced to unity, we easily get the recent results obtained by Srivastava, Saigo and Raina [18, Theorems (3) and (4)].

A further application concerning a number of technical problems involving wave motion (cf. [8, p. 16, Sect. 1.31]) can be considered by applying Corollary 2 to the function $\psi(x)$ defined by

$$
\psi(x)=f\left(a \sqrt{\left.x^{2}-b^{2}\right)} H(x-b) \quad(a>0 ; b>0)\right.
$$

where $H(x)$ denotes the Heaviside unit function. So, we find from Corollary 2 that

$$
R^{*}\left[s^{\gamma} L\{\psi(x) ; s\}\right]=\Gamma(\mu) \sum_{e, p, u, \nu} \phi(e, p, u, \nu) z^{w} s^{\gamma} \int_{b}^{\infty} \psi(x) V(\mu, \eta, \lambda, \gamma, s) d x
$$

for $\operatorname{Re}(\gamma+\eta+\rho w)>\max (0, \operatorname{Re}(\eta-\lambda))-1, \operatorname{Re}(s)>0$, and

$$
K^{*}\left[s^{\gamma} L\{\psi(x) ; s\}\right]=\Gamma(\mu) \sum_{e, p, u, \nu} \phi(e, p, u, \nu) z^{w} s^{\rho w} \int_{b}^{\infty} \psi(x) U(\mu, \delta, \lambda, \gamma ; x, \cdot) d x
$$

for $\operatorname{Re}(\mu)>0, \operatorname{Re}(s)>0$ and non integer values of $(\lambda-\delta),(\gamma-\delta-\rho w)$ and $(\gamma-\lambda-\rho w)$, and $U$ and $V$ being given by (3.17) and (3.19) respectively.

If we reduce the generalized polynomial set in $(3.25)$ and $(3.26)$ to unity, we get the results established by Srivastava, Saigo and Raina [18, p. 9, Eqs (4.2) and (4.3)]. Further, if we set $\gamma=0$ and $\delta=-\mu$ in (3.26) and the generalized polynomial set reduced to unity, we get a result obtained by Agal and Koul [1]. 


\section{Acknowledgement}

The authors are extremely grateful to Prof. H. M. Srivastava, University of Victoria, Canada, for making certain valuable suggestions for the improvement of this paper. The authors (K.C.G.) and (T.O.S) are also thankful to University Grants Commission, New Delhi, and AL-Azhar University of Gaza, Palestine, respectively, for providing the necessary financial support to carry out this work.

\section{References}

[1] S. N. Agal and C. L. Koul, "Weyl fractional calculus and Laplace transform," Proc. Indian Acad. Sci. (Math. Sci.), 92(1983), 167-170.

[2] B. D. Agrawal and J. P. Chaubey, "Operational derivation of generating relations for generalized polynomials," Indian J. Pure Appl. Math., 11(1980), 1155-1157; ibid. 11 (1981), 357-379.

[3] R. Agrawal, R. S. Pareek and M. Saigo, "A general fractional integral formula," J. Fractional Calculus, 7(1995), 55-60.

[4] S. K. Chatterjea, "Qulques fonctions generatrices des polynomes d'Hermite, du point de vue de lalgebre de Lie," C. R. Acad. Sci Paris Ser. A-B, 268(1969), A 600-602.

[5] H. W. Gould and A. T. Hopper, "Operational formulas connected with two generalizations of Hermite polynomials," Duke Math. J., 29(1962), 51-63.

[6] K. C. Gupta and R. C. Soni, "On unified fractional integral operators," Proc. Indian Acad. Sci. (Math. Sci.), 106(1996), 53-64,

[7] H. L. Krall and O. Frink, "A new class of orthogonal polynomials: the Bessel polynomials," Trans. Amer. Math. Soc., 65(1949), 100-115.

[8] N. W. McLachlan, Modern Operational Calculus with Applications in Technical Mathematics, Macmillan, London, 1948.

[9] S. K. Raizada, A study of unified representation of special functions of mathematical physics and their use in statistical and boundary value problems, $\mathrm{Ph}$. D thesis, Bundlkhand Univ., India, 1991.

[10] M. Saigo, "A remark on integral operators involving the Gauss hypergeometric functions," Math. Rep. College General Ed. Kyushu Univ., 11(1978), 135-143.

[11] M. Saigo and R. K. Raina, "Fractional Calculus operators associated with a general class of polynomials," Fukuoka Univ. Sci. Rep., 18(1988), 15-22.

[12] M. Saigo, S. P. Goyal and S. Saxena, "A theorem relating a generalized Weyl fractional integral, Laplace and Varma transforms with applications," J. Fractional Calculus, 13(1998), 43-56.

[13] R. K. Saxena and R. K. Kumbat, "Integral operators involving H-function," Indian J. pure Appl. math., 5(1974), 1-6.

[14] R. K. Singh and K. N. Shrivastava, "A note on generalization of Laguerre and Humbert polynomials," Ricerca (Napoli), 14 (2)(1963), 11-21, Errata, Ibid, 15(1964), 63.

[15] H. M. Srivastava and H. L. Manocha, A Treatise on Generating Functions, Wiley/Halsted, New York, 1984.

[16] H. M. Srivastava, K. C. Gupta and S. P. Goyal, The H-Functions of One and Two Variables with Applications, Sourth Asian Pubishers, New Delhi-Madras, 1982. 
[17] H. M. Srivastava and R. Panda, "on the unified presentation of certain classical polynomials," Boll. Un. Mat. Ital., 12(4)(1975), 306-314.

[18] H. M. Srivastava, M. Saigo and R. K. Raina, "Some existence and connection theorems associated with the Laplace transform and a certain class of integral operators," J. Math. Anal. Appl., 172(1993), 1-10.

Emeritus Fellow (U.G.C.), M.R. Engineering College, Jaipur 302017, India.

Department of Mathematics, University of Rajasthan, Jaipur 302004, Indian. 